\title{
Dendritic cell-derived exosomes for cancer therapy
}

\author{
Jonathan M. Pitt, ${ }^{1,2,3}$ Fabrice André, ${ }^{1,3,4,5}$ Sebastian Amigorena, ${ }^{6}$ Jean-Charles Soria, ${ }^{1,3,4,7}$ Alexander Eggermont, ${ }^{1}$ \\ Guido Kroemer, $8,9,10,11,12,13,14$ and Laurence Zitvogel ${ }^{1,2,3,6,15}$
}

IInstitut de Cancérologie Gustave Roussy Cancer Campus (GRCC), Villejuif, France. ${ }^{2}$ INSERM Unit U1015, Villejuif, France. ${ }^{3}$ Université Paris Sud, Université Paris-Saclay, Faculté de Médecine, Le Kremlin Bicêtre, France. ${ }^{4}$ INSERM Unit U981, Villejuif, France. ${ }^{5}$ Department of Medical Oncology, Villejuif, France. ${ }^{6}$ INSERM Unit U932, Institut Curie, Paris, France. ${ }^{7}$ Drug Development Department (DITEP), Villejuif, France. ${ }^{8}$ INSERM U848, Villejuif, France. ${ }^{9}$ Metabolomics Platform, CRCC, Villejuif, France. ${ }^{10}$ Equipe 11 labellisée Ligue contre le Cancer, Centre de Recherche des Cordeliers, INSERM U1138, Paris, France. "11Pôle de Biologie, Hôpital Européen Georges Pompidou, Paris, France. ${ }^{12}$ Université Paris Descartes, Sorbonne Paris Cité, Paris, France. ${ }^{13}$ Université Pierre et Marie Curie, Paris, France. ${ }^{14}$ Karolinska Institute, Department of Women's and Children's Health, Karolinska University Hospital, Stockholm, Sweden.

${ }^{15}$ Center of Clinical Investigations in Biotherapies of Cancer (CICBT) 507, Villejuif, France.

\begin{abstract}
DC-derived exosomes (Dex) are nanometer-sized membrane vesicles that are secreted by the sentinel antigen-presenting cells of the immune system: DCs. Like DCs, the molecular composition of Dex includes surface expression of functional MHC-peptide complexes, costimulatory molecules, and other components that interact with immune cells. Dex have the potential to facilitate immune cell-dependent tumor rejection and have distinct advantages over cell-based immunotherapies involving DCs. Accordingly, Dex-based phase I and II clinical trials have been conducted in advanced malignancies, showing the feasibility and safety of the approach, as well as the propensity of these nanovesicles to mediate T and NK cell-based immune responses in patients. This Review will evaluate the interactions of Dex with immune cells, their clinical progress, and the future of Dex immunotherapy for cancer.
\end{abstract}

\section{Introduction}

As the sentinel antigen-presenting cells (APCs) of the immune system, DCs play a central role in initiating antigen-specific immunity and tolerance (1). In cancer, DCs act as the initial link between oncogenesis and the host immune system, the first step of a cancer/immunity cycle that aims to eliminate cancer cells through the activation of T cells (2). Tumor-proximal DCs can capture neoantigens created and released during oncogenesis, which the DCs subsequently process and present to cognate $\mathrm{T}$ cells to generate antitumor $\mathrm{T}$ cell responses. However, such $\mathrm{T}$ cell responses can only be generated if certain additional conditions are met in the local environment (2). These conditions consist of locally present immunogenic signals, such as proinflammatory cytokines, danger-associated molecular patterns (DAMPs), or pathogenassociated molecular patterns (PAMPs). Such signals trigger DCs to present captured tumor-associated antigens (TAAs) via MHC class I (MHC-I) and MHC-II molecules to T cells in cooperation with costimulatory molecules such as CD80 and CD86, resulting in the priming and activation of TAA-specific effector T cells.

Therapies harnessing these properties of DCs to generate immune responses against tumors have great potential, though clinical progress of this application remains in its infancy. One notable exception is the success of the immunotherapy sipuleucel-T for early-stage, hormone-refractory prostate cancer. Sipuleucel-T is composed of autologous peripheral blood mononuclear cells (PBMCs) including APCs (such as DCs and their precursors) that have been stimulated ex vivo with a fusion protein consisting of the cytokine granulocyte macrophage colony-stimulating factor

Conflict of interest: The authors have declared that no conflict of interest exists. Reference information: J Clin Invest. 2016;126(4):1224-1232. doi:10.1172/JCI81137.
(GM-CSF), which drives DC differentiation and activation, combined with a prostate antigen (3). Nonetheless, DC-based immunotherapy is challenging to practice in clinical settings. Implementing such therapies across large populations is costly, requires dedicated expertise, and requires monitoring of well-defined quality control parameters. Furthermore, it is difficult to store DCs over long periods of time while maintaining their efficacy (4).

The use of DC-derived exosomes (Dex) has been heralded as a solution to many of the technical challenges associated with DC-based immunotherapy (see Table 1) because they maintain the essential immunostimulatory faculties of DCs (e.g., sharing the ability to present antigens to $\mathrm{T}$ cells), while the stable nature of exosomal membranes allows their frozen storage for at least 6 months (5). As biologics, Dex are also more amenable to a strictly regulated and monitored manufacturing process (e.g., their composition and MHC-I and MHC-II content can be easily defined), and they lack the risks associated with viable cellular or viral therapies such as the risk of in vivo replication (6). Finally, treatment with cell-free Dex may be more resistant to immunomodulatory events that occur in tumors than other anticancer vaccines; such events can downregulate costimulatory molecules on DCs and impede stimulation of $\mathrm{T}$ cell responses (7).

As discussed in detail in other sections of this review series, DCs are one of the many cell types able to secrete membrane vesicles, such as exosomes, into the extracellular environment. This manner of signaling can modulate recipient cells, such as immune cells or cancer cells, to a level beyond classical ligand/receptor signaling pathways and can create complex cellular modifications that may play a substantial role in how tumor development or immune responses proceed. Moreover, detection of circulating, cancer cell-derived exosomes can serve as a noninvasive diagnostic and screening tool to detect early stages of cancer, facilitating 
Table 1. Potential functional differences between DC- and Dex-based therapy

Practical/functional difference

Determination of molecular parameters for QC

Abundance of surface peptide-MHC-II complexes

Stability of vaccine preparation

Immunostimulation of NK cells

Localization

Resistance to immunosuppression
DC

Molecular composition can change,

difficulties defining $\mathrm{Q} C$ parameters

Fewer peptide-MHC-II complexes on the surface membrane, lower yields

Living cell, issues with storage and stability over longer periods

$D C s$ can fail to express sufficient NK cell receptor ligands (42)

Rely on chemokine gradients and other signals for access and localization in lymph nodes (54)

Susceptible to immunosuppressive molecules and immunoregulation in the tumor microenvironment (7)
Dex

Dex molecular composition can be strictly defined for each patient (5)

Dex are enriched in peptide-MHC-II complexes, by $10-100 \times$ that of DCs, allowing up to 6 months Dex therapy from a single leukapheresis (5)

Lipid composition of Dex allows high stability for $>6$ months at $-80^{\circ} \mathrm{C}(5)$

Membrane expression of ligands for NK receptors

$(42,43,45)$

Potentially more thorough dispersion within lymph nodes and access to multiple immune cell types (74), not reliant on chemotactic signaling but rather on their surface membrane receptor topography $(12,75,76)$

Cannot respond to immunosuppressive molecules

QC, quality control.

deployment of therapeutic interventions (8) (discussed elsewhere in this review series; refs. 9, 10,11). Secreted membrane vesicles consist of a surrounding lipid bilayer containing various transmembrane proteins; the bilayer encloses various cytosolic components and molecules (e.g., proteins, lipids, and nucleic acids) from the donor cell. While several different subtypes of membrane vesicles have been characterized, such as apoptotic bodies and the microvesicles that directly bud from the plasma membrane (12), the focus of this review will be on the exosomes secreted by DCs, which have progressed to the stage of clinical testing.

\section{The molecular composition of Dex}

The molecular composition of Dex allows functionality as an immunotherapy. Similarly to all other exosomes, Dex have a size of 30-100 $\mathrm{nm}$ and are initially formed within the cell by the inward budding of endosomal membranes. The resulting endosome formed by this process with its content of intraluminal vesicles is referred to as a multivesicular body (MVB) (13). If MVBs are not targeted for lysosomal degradation, they may fuse with the cell surface membranes of DCs to allow release of their intraluminal vesicles as exosomes $(13,14)$. The first studies of the Dex proteome revealed a unique molecular composition that allowed for strong immunostimulatory functionality $(15,16)$. Dex were found to possess MHC-I and MHC-II molecules, which could potentially stimulate $\mathrm{CD}^{+}$and $\mathrm{CD} 4^{+} \mathrm{T}$ cells, respectively, as well as costimulatory molecules (15-18) (see below). Dex possess a variety of surface membrane proteins, including the integrin $\alpha$ and $\beta$ chains ( $\alpha \mathrm{M} \beta 2)$, the immunoglobulin family member ICAM-1, and milk fat globule EGF factor 8 (MFG-E8), which allow for effective targeting and docking to recipient cells $(12,15,16)$. MFG-E8 is an abundant protein constituent of Dex membranes that can bind externalized phosphatidylserine on the Dex outer membrane and facilitate uptake of exosomes by linking Dex with $\alpha v \beta 3$ or $\alpha v \beta 5$ integrins present on the receiving cell (16). The tetraspanin family of proteins, including CD9, CD63, and CD81, are also well-defined constituents of the Dex surface membrane $(15,16)$. These and other membrane microdomain organizing proteins are postulated to participate in exosome/acceptor cell interactions $(15,16)$.

Various cytosolic proteins derived from the donor DC are present on the Dex outer membrane. These proteins appear to be related to Dex biogenesis from DC endocytic compartments (e.g., annexins, RAB proteins, and tumor susceptibility gene 101 [TSG101]) or are participants in signal transduction pathways (e.g., G proteins and kinases). The heat shock cognate protein HSC73 makes up a significant fraction of the cytosolic proteins found in Dex (16). This HSP70 family member, together with members of the HSP90 family that are also present within Dex, may add to Dex immunogenicity, since these proteins can activate various immune cells and have significant roles as antigen chaperones and in MHC loading (18). In addition to these protein families, exosomes also contain mRNA and small RNA (including miR) molecules, which they can transfer between cells $(19,20)$. RNA transfer via Dex has been shown as a means of communication and posttranscriptional modification between DCs, since exosome-delivered miRs were demonstrated to repress target mRNAs of Dex-accepting DCs (21). These findings could have wide implications for Dex immunotherapy, as they suggest that particular RNA profiles of Dex (or, more specifically, those of the donor DC) could influence Dex immunogenicity through posttranscriptional modifications of acceptor APCs.

Direct and indirect molecular interactions between Dex and $T$ cells. Perhaps the most important attributes of Dex surface membranes, which also set them apart from exosomes secreted by other immune cells, is their possession of DC-originating molecules involved in antigen processing and presentation. It was initially discovered that exosomes derived from B cells possess functional peptide/MHC-II complexes upon their surface membranes and that they could induce antigen-specific MHC-II-restricted T cell responses (22). Dex also have abundant MHC-I molecules (16, $14)$, which are similarly able to induce $\mathrm{T}$ cell responses $(23,24)$. Importantly, Dex derived from tumor peptide-stimulated DCs were able to prime tumor-specific cytotoxic T lymphocyte (CTL) 


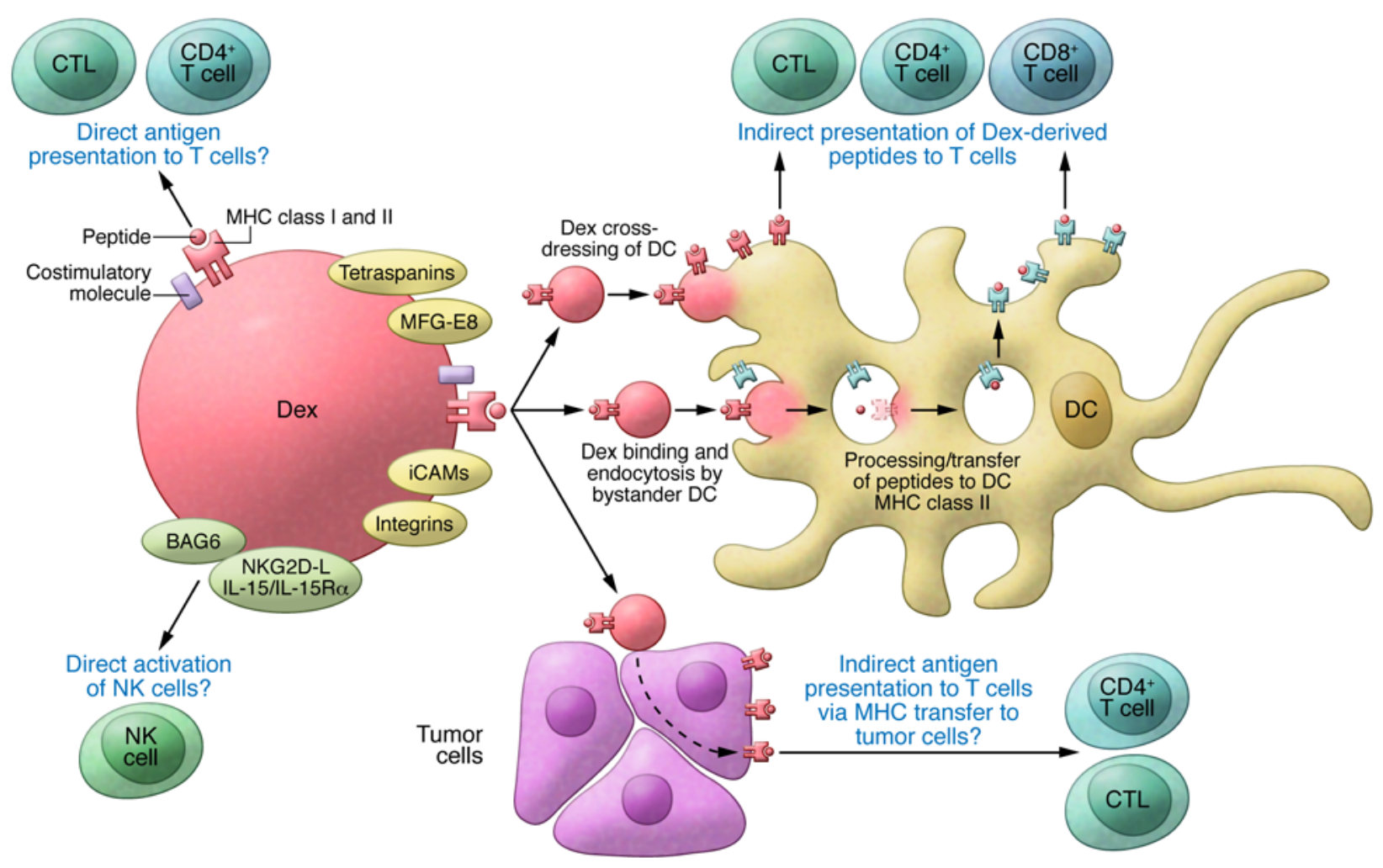

Figure 1. Dex interactions with immune cells. Dex may stimulate T cells by direct and indirect routes. The presence of MHC-I and MHC-II molecules on the surface of Dex gives them the potential to directly stimulate CTLs and CD4 ${ }^{+} \mathrm{T}$ cells, respectively. Dex surface costimulatory molecules aid this process. A more likely route for Dex stimulation of T cells occurs indirectly via bystander DCs through two possible mechanisms. The first involves Dex internalization and transfer of antigenic peptides to MHC molecules of the DC. These MHC/peptide complexes may then be transported to the DC surface for presentation to T cells. The second mechanism involves Dex transfer of $\mathrm{MHC} /$ peptide complexes directly to the $\mathrm{DC}$ surface, a process termed cross-dressing. It has been suggested that Dex may also transfer MHC/peptide complexes to tumor cell surfaces, enabling tumor cell targeting by host T cells. Additionally, Dex have been shown to possess BAG6, NKG2D-L, and the IL-15/IL-15R $\alpha$ complex, which can each result in NK cell activation.

responses in vivo, and a single intradermal injection of this preparation resulted in tumor growth delay or, in some cases, a complete eradication of established murine tumors (17). The presence of the potent costimulatory molecule CD86 on the surface of Dex may also have contributed to $\mathrm{T}$ cell priming and activation (17).

Several mechanisms have been proposed for how Dex present antigens via their MHC molecules to stimulate $\mathrm{T}$ cell responses (Figure 1), presumably within lymph nodes. Dex have been shown to stimulate $\mathrm{T}$ cells directly in vitro, though it appears that this mechanism operates most efficiently as a restimulation of activated $\mathrm{T}$ cells, memory $\mathrm{T}$ cells, or $\mathrm{T}$ cell lines, clones, and hybrids $(14,22,23)$. Direct Dex-to-T cell stimulation appears to be inefficient in priming naive $\mathrm{T}$ cells (25) and is unlikely to occur to a significant extent in vivo (26). Furthermore, Dex appear to be less efficient in directly stimulating $\mathrm{T}$ cell responses when compared with the T cell stimulatory capacity of the donor DCs (27), but stimulation may be improved if Dex are immobilized or if their concentration is increased in vitro $(14,27)$.

Rather than a direct Dex/T cell route of stimulation, evidence suggests that Dex or other APC-derived exosomes stimulate T cell responses far more efficiently via indirect antigen presentation through transfer of antigenic peptide/MHC complexes to bystander APCs (Figure 1 and refs. 14, 27-30). Furthermore, priming of naive $\mathrm{T}$ cells has been shown to occur only if APCs are present $(25,26,28)$.
The constituents of exosome surface membranes, such as integrins and ICAMs, facilitate their binding to and uptake by APCs to enable indirect antigen presentation. Interestingly, the efficiency of indirect $\mathrm{T}$ cell stimulation appears to be highly dependent upon the activation status of the donor APC from which exosomes are derived. For example, the exosomes released from DCs treated with LPS or IFN- $\gamma$ (mature DCs) have increased surface expression of ICAM-1 that presumably enhances their uptake by DCs and increased expression of MHC molecules and CD86, which contribute to T cell activation (31-33). Consistent with their topography, these mature exosomes have a greater capacity to stimulate $\mathrm{T}$ cells compared with exosomes derived from immature DCs (24, 31-33).

Following binding to bystander APCs, some of the Dex - but not all individual Dex molecules - are internalized by phagocytosis or macropinocytosis, with the remaining Dex likely retained on the DC surface. The proportion of internalized exosomes is dependent upon the maturation status of the accepting APC. It has been shown that immature DCs internalize exosomes more efficiently than mature DCs, whereas mature DCs are likely to retain more Dex on their surfaces, although presumably surface-retained Dex still interact with T cells (26). Following internalization, Dex peptide/MHC complexes can be reprocessed via endosomal pathways within the APC, resulting in the transfer of antigenic peptides from Dex MHCs to APC MHCs $(14,26,34)$. These MHC/ 
Table 2. Dex clinical trials

\begin{tabular}{|c|c|c|c|c|c|c|c|}
\hline $\begin{array}{l}\text { Patient } \\
\text { Population }\end{array}$ & Phase & $n$ & $\begin{array}{c}\text { Dex format and } \\
\text { approach }\end{array}$ & TAAs & Effect on immune cell parameters & Trial Outcome & Ref. \\
\hline $\begin{array}{l}\text { Advanced } \\
\text { NSCLC }\end{array}$ & I & $\begin{array}{l}13 \text { (9 } \\
\text { completed } \\
\text { therapy) }\end{array}$ & $\begin{array}{l}\text { Dex, } 4 \text { vaccinations } \\
\text { at weekly intervals }\end{array}$ & MAGE peptides & $\begin{array}{l}\text { Limited T cell reactivity. DTH } \\
\text { response in } 3 / 9 \text { patients. Possible } \\
\text { increase in Treg functions and } \\
\text { some NK cell lytic activity. }\end{array}$ & $\begin{array}{l}\text { Safe, well tolerated. Stability of disease } \\
\text { in } 2 \text { patients with initial disease } \\
\text { progression, stability of disease of }>12 \\
\text { months in } 2 / 4 \text { initially stable patients. }\end{array}$ & 38 \\
\hline $\begin{array}{l}\text { Metastatic } \\
\text { Melanoma }\end{array}$ & I & 15 & $\begin{array}{l}\text { Dex, } 4 \text { vaccinations } \\
\text { at weekly intervals }\end{array}$ & MAGE peptides & $\begin{array}{l}\text { No MAGE-specific T cell } \\
\text { reactivity. No DTH response. } \\
\text { NK cell activation. }\end{array}$ & $\begin{array}{c}\text { Safe, well tolerated. } 2 \text { stable diseases, } \\
1 \text { minor response, } 1 \text { partial response, } \\
1 \text { mixed response. }\end{array}$ & 37,42 \\
\hline $\begin{array}{l}\text { Advanced } \\
\text { colorectal } \\
\text { cancer }\end{array}$ & I & 40 & $\begin{array}{l}\text { Aex prepared from patient } \\
\text { ascites } \pm \mathrm{GM}-\mathrm{CSF}, \\
4 \text { vaccinations at } \\
\text { weekly intervals }\end{array}$ & $\begin{array}{l}\text { CEA detected in Aex } \\
\text { (inherent loading) }\end{array}$ & $\begin{array}{l}\text { Aex+CM-CSF induced CEA-specific } \\
\text { CTL responses. DTH induction } \\
\text { in both groups. }\end{array}$ & $\begin{array}{l}\text { Safe, well tolerated. } 1 \text { stable } \\
\text { disease and } 1 \text { minor response, } \\
\text { both in Aex+GM-CSF group. }\end{array}$ & 40 \\
\hline $\begin{array}{l}\text { Advanced } \\
\text { NSCLC }\end{array}$ & $\|$ & $\begin{array}{l}26(22 \\
\text { completed } \\
\text { therapy) }\end{array}$ & $\begin{array}{l}\text { IFN- } \gamma \text {-derived Dex, } 4 \text { weeks } \\
\text { of weekly maintenance } \\
\text { immunotherapy after CTX }\end{array}$ & $\begin{array}{c}\text { MAGE-A1, MAGE-A3, NY-ESO, } \\
\text { Melan-A/MART1 (all MHC-I- } \\
\text { restricted peptides); MAGE-A3, } \\
\text { EBV (MHC-II-restricted peptides) }\end{array}$ & $\begin{array}{l}\text { Limited T cell activity. Increased } \\
\text { NK cell functions correlating } \\
\text { with longer PFS. }\end{array}$ & $\begin{array}{l}7 \text { patients }(32 \%) \text { with stable } \\
\text { disease }>4 \text { months. } 1 \text { patient } \\
\text { had a grade- } 3 \text { hepatotoxicity. }\end{array}$ & 39 \\
\hline
\end{tabular}

EBV, Epstein-Barr virus.

peptide complexes can then be transported back to the DC surface for presentation to $\mathrm{T}$ cells.

A second mechanism for Dex-mediated indirect antigen presentation to $T$ cells is a process known as cross-dressing where, following binding to APCs, Dex merge with the acceptor APC surface membrane, thereby transferring their peptide/MHC complexes (Figure 1 and refs. 28, 35). This would potentially allow immediate recognition of MHC-presented peptides by T cells without the need for antigen processing and could enable the Dex-transferred antigen presentation machinery to benefit from additional costimulatory molecules and factors present on the accepting APC. In support of this paradigm, Dex can activate $\mathrm{T}$ cells only if mature CD8 $\alpha^{-}$DCs are present, even when mature DCs are deficient for MHC-II (28), suggesting Dex-to-DC cross-dressing of MHC/peptide complexes. Expression of the costimulatory molecules CD80 and CD86 on the presenting DCs was required for T cell activation (28). However, Dex MHC-I cross-dressing of bystander DCs does not appear to induce ovalbumin-specific (OT-I) $\mathrm{CD} 8^{+} \mathrm{T}$ cell proliferation in an ovalbumin experimental model but instead occurs through Dex internalization and reprocessing of Dex-derived antigen onto MHC molecules of the recipient DC (30).

A third indirect route through which Dex could activate $\mathrm{T}$ cells may occur via tumor cells. In a recent study, treatment of human breast adenocarcinoma cells with Dex was sufficient to restimulate previously primed $\mathrm{T}$ cells, inducing significantly higher percentages of IFN- $\gamma$-secreting T cells compared with exposure to non-Dex-treated adenocarcinoma cells (36). This suggests that incorporation of Dex by tumor cells can potentially convert tumor cells into stronger immunogenic targets, opening a new avenue of therapeutic strategies to increase immune targeting of tumors.

\section{Clinical trials of Dex immunotherapies}

In view of the high potential and benefits of Dex as an immunotherapy, Dex have been developed for clinical use as cell-free cancer vaccines. Two phase I clinical trials $(37,38)$ and one phase II trial (39) using Dex have now been completed in advanced cancer patients (see Table 2).

Technical considerations. The manufacturing steps for Dex preparation and purification are detailed in Figure 2. More than 100 individual Dex preparations have been made so far, as the process of vaccine generation is feasible from cells derived from a single leukapheresis. Quality control parameters include the determination of tetraspanins (CD81, CD82, and CD63), high levels of HLA-DR, costimulatory molecules, and exosomal hallmarks such as TSG101 and HSP70 (31). Verification of peptide loading can be performed by incubating Dex with or without HLA-A2- APCs (DCs) and a peptide-specific T cell clone. In this setting, indirect Dex loading of MHC-I-restricted peptides (i.e., loaded onto the donor IFN- $\gamma$-treated DCs on day 4 of culture) appears to be as effective as direct peptide loading for stimulation of antigenspecific $\mathrm{T}$ cell clones when short peptides were loaded (31). Activation of the latter relative to negative controls confirms functional $\mathrm{T}$ cell stimulatory capacity of Dex. For the phase II trial, Dex batch release depended upon a high ratio of tetraspanins and HLA-DR (MHC-II) when compared with control $(31,39)$. In the phase I trials, Dex MHC-I natural epitopes were acid eluted, whereas no prior elution was performed in the Dex phase II trial. This discontinuation of peptide elution was based on the capacity of high-affinity peptides to compete against and/or coexist with natural epitopes on exosomal surfaces, as shown using ex vivo functional assays with melan-A-specific (MART1-specific) CTL clones $(31,39)$.

Phase I trials. The initial phase I trials used Dex produced from autologous monocyte-derived DC cultures loaded with antigenic HLA-presented peptides of melanoma-associated antigen (MAGE). The patients in the two studies received four vaccinations with Dex at weekly intervals.

In the first of these phase I studies (38), nine patients with MAGE-expressing pretreated advanced non-small cell lung cancers (NSCLC) were eligible to receive Dex therapy (Table 2). Three patients who did not exhibit reactivity to MAGE prior to immuni- 


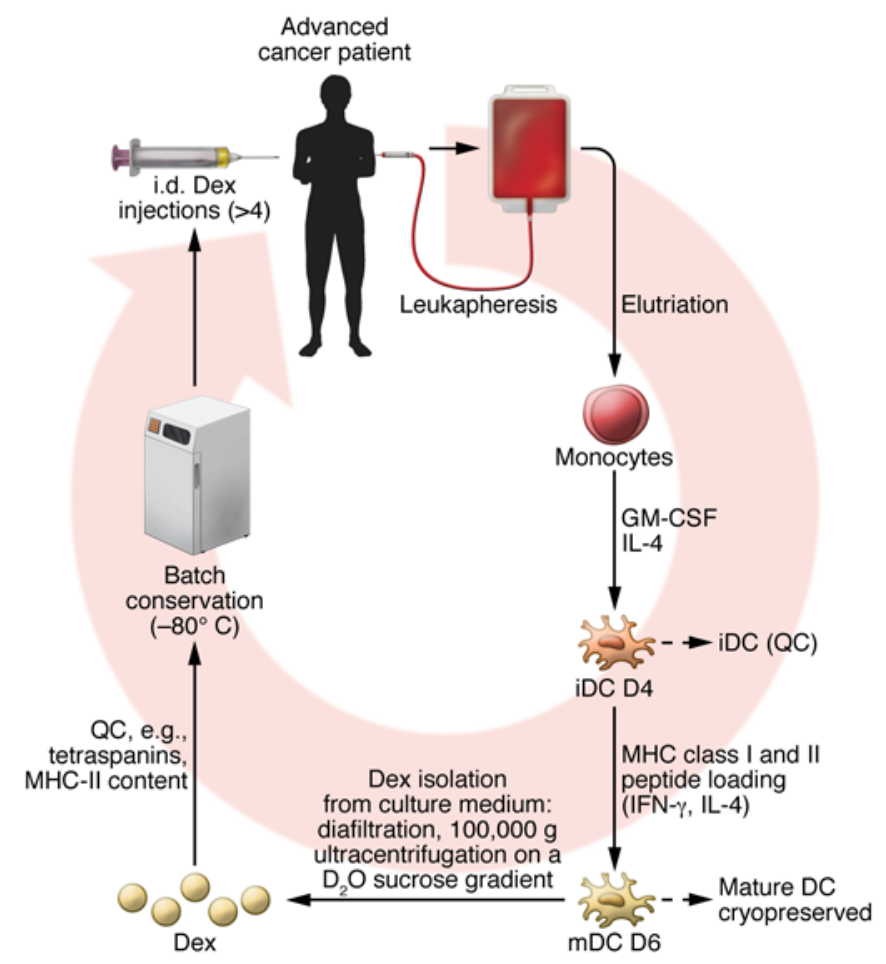

zation showed systemic MAGE-sensitive immune responses one week following the last Dex injection, as determined by delayedtype hypersensitivity (DTH) response. Increases in T cell activity were detected by enzyme-linked immunospot (ELISPOT) assay to MAGE peptides in one of five tested patients. Treg suppression was postulated as a reason for this low rate of $\mathrm{T}$ cell activation, since Tregs (defined as $\mathrm{CD} 4{ }^{+} \mathrm{CD} 25^{+} \mathrm{T}$ cells) as a percentage of total $\mathrm{CD} 4^{+} \mathrm{T}$ cells were increased in two of three patients after Dex therapy compared with baseline levels. Interestingly, a potential increase in NK cell lysis ability was also observed in two of four analyzable samples. A good safety profile was the main clinical outcome for the NSCLC phase I study, with disease stability observed in two patients who had disease progression at the start of the study and continued disease stability over 12 months in two of four initially stable patients (38).

The second phase I study enrolled 15 patients with MAGE3 ${ }^{+}$ advanced malignant melanoma (MM), all of whom received Dex (Table 2 and ref. 37). Of these patients, one patient exhibited a partial response to Dex immunotherapy and developed a halo of depigmentation around naevi and disappearance of arterial neovasculature, concomitant with tumor shrinkage. This patient was given continuation therapy with Dex for 4 months, during which time there was stabilization of disease without toxicity. Disease stabilization for up to 24 months was also achieved for another patient who received continued Dex therapy. The overall clinical outcome was two stable diseases, one minor response, one partial response, and one mixed response at skin or lymph node sites, with some of these responses occurring in patients with progressive disease who had previously received other biotherapies or alternative antitumor vaccinations. However, similarly to the other trial, neither MAGE-specific $\mathrm{CD}^{+}$and $\mathrm{CD}^{+}{ }^{+} \mathrm{T}$ cell responses nor DTH responses could be detected in peripheral blood (37).
Figure 2. The GMP manufacturing of Dex immunotherapy. Leukapheresis is performed for advanced cancer patients who will undergo Dex therapy. Within a cell therapy unit GMP (good manufacturing practices) laboratory, monocytes are isolated following elutriation, and these are differentiated to immature DCs (iDC) by addition of GM-CSF and IL-4 in culture. iDCs may then undergo a quality control (QC) check before loading of MHC-I- and MHC-II-binding peptides in the presence of IFN- $\gamma$ to generate mDC. Peptide-loaded exosomes from culture supernatants can then be isolated and concentrated by a process of centrifugation, diafiltration, and finally ultracentrifugation over $\mathrm{a}_{2} \mathrm{O}$ sucrose gradient. QC testing of immunological characteristics (e.g., tetraspanin content of Dex, MHC-II and costimulatory molecule levels) and immunostimulatory capacity (e.g., ability to stimulate a peptide-cognate T cell clone) is then performed for each preparation to determine whether a given batch can be released. Released batches may then be stored $\left(-80^{\circ} \mathrm{C}\right)$ for therapeutic administration through intradermal (i.d.) injections. Production of Dex for immunotherapy takes approximately three weeks from the initial leukapheresis (this includes time for Dex manufacturing, QC testing, and treatment of the patient with metronomic cyclophosphamide).

A third phase I clinical trial used ascites-derived exosomes (Aex) in combination with GM-CSF to treat 40 advanced colorectal carcinoma (CRC) patients with Aex alone or Aex plus GM-CSF. The patients received four immunizations at weekly intervals (Table 2 and ref. 40). Exosomes were prepared from malignant ascites of the patients and were found to be enriched in MHC-I and MHC-II molecules, costimulatory molecules, and ICAMs. They were also shown to contain the immunogenic carcinoembryonic antigen (CEA) of CRC. A good safety profile was observed for Aex; however, no detectable therapeutic responses were observed except in one patient with stable disease and a second patient who exhibited a minor response after treatment with Aex plus GM-CSF. Interestingly, and in contrast with the low-level stimulation of $\mathrm{T}$ cell responses in the Dex clinical studies, Aex plus GM-CSF could induce CEA-specific antitumor CTL responses (40). The majority of the Aex were believed to be derived from CRC cells, although the ascites used in this study likely contained other immune or mesothelial cell-derived nanoparticles or microvesicles. A greater level of TAAs present in Aex compared with Dex may have also been responsible for the greater $\mathrm{T}$ cell responses observed in this study as compared with the two Dex phase I trials. However, given findings that tumor-derived exosomes can possess immunosuppressive properties and can facilitate tumor growth, metastasis, and development of drug resistance, care should be taken to distinguish the exact exosome composition of Aex and similar preparations, with a view to removing potentially adverse tumorderived exosomes when such technologies become available $(8,41)$.

Interpretation of the immunological findings from the phase I trials: Dex stimulate NK cell functions. The phase I studies confirmed the safety of Dex administration in patients and highlighted the feasibility of large-scale Dex production. Somewhat surprisingly, given the encouraging results from preclinical studies, Dex were 
apparently poor stimulators of $\mathrm{T}$ cell responses. However, Dex were found to stimulate NK cells.

Following the MM phase I trial, it has been shown that Dex derived from human immature DCs possess killer cell lectin-like receptor subfamily K, member 1 ligands (NKG2D-L) upon their surface, which can directly engage NKG2D present on NK cells, leading to their activation (42). By using samples from the MM clinical trial, it was found that the number of circulating NK cells significantly increased after 4 weekly vaccinations with Dex. Additionally, NKG2D expression levels and cytotoxicity of NK cells were restored after Dex vaccination in $50 \%$ of MM patients presenting with NK cell defects at diagnosis (42). Further investigations of Dex/NK cell interactions in vivo revealed that Dex promote IL-15R $\alpha$-dependent NK cell proliferation and an NKG2D-mediated activation of NK cells. These Dex-mediated pro-NK cell effects coincided with improved metastatic control by NK1.1 ${ }^{+}$cells in C57BL/6 mice inoculated i.v. with B16F10 melanoma cells (42). Dex derived from immature human DCs (and exosomes derived from 293T cells) also express BCL2-associated athanogene 6 (BAG6, also known as BAT3) on their surface (43), which has been identified as a ligand for natural cytotoxicity triggering receptor 3 (NKp30) receptors on NK cells (44). Exosomal BAG6 expression levels were shown to directly correlate with NK cell cytokine release (43). Additionally, Dex surface expression of TNF induces NK cell IFN- $\gamma$ production (45).

Phase II trial. The poor capacity of Dex to stimulate $\mathrm{T}$ cell responses in the initial phase I studies encouraged new approaches to improve the interactions of Dex with the adaptive arm of the host immune response. As previously described, the use of exosomes derived from LPS- or IFN- $\gamma$-matured DCs (mDex) has been one such new approach, following discoveries that $\mathrm{mDex}$ can induce greater $\mathrm{T}$ cell stimulation than Dex derived from immature DCs $(24,31,33)$. Application of these findings to human DC cultures led to a clinical-grade manufacturing process for Dex vaccines (31) in which IFN- $\gamma$ stimulation of human DC cultures induces the expression of costimulatory molecules and ICAMs on Dex, yielding a second-generation Dex with enhanced immunostimulatory properties $(31,46)$. A phase II clinical trial was launched using second-generation Dex in advanced NSCLC patients, with the aim of investigating whether IFN- $\gamma$-Dex used as maintenance immunotherapy can ameliorate the rate of progression-free survival (PFS) at 4 months after platinum-based chemotherapy (39).

Twenty-two advanced NSCLC patients who had received four cycles of a first-line platinum-based chemotherapy were eligible to receive IFN- $\gamma$-Dex (39). Patients were administered metronomic oral cyclophosphamide (CTX) ahead of IFN- $\gamma$-Dex maintenance therapy based upon preclinical (47-49) and clinical evidence (48, 50) demonstrating that this regimen reduces Treg function and stimulates dual IFN- $\gamma /$ IL-17-producing T cells, thereby facilitating Dex-mediated $\mathrm{T}$ cell priming and restoring $\mathrm{T}$ cell and $\mathrm{NK}$ cell functions. Seven patients (32\%) exhibited stable disease after nine injections with IFN- $\gamma$-Dex, and these patients continued receiving Dex therapy every three weeks. Consequently, the primary endpoint of the trial, a PFS of $50 \%$, was not reached. No objective response was recorded in the trial, although one patient experienced a long-term stabilization that allowed tumor debulking surgery, a downgrading of disease status by pathologists, and eligibility for local adjuvant thoracic and vertebral radiotherapy.
Regarding the immunological readouts, IFN- $\gamma$-Dex immunotherapy again failed to show evidence of TAA-specific T cell immune responses in the patient cohort (39) despite multiepitope loading and the CTX adjuvant. The immunostimulatory capacity of IFN- $\gamma$-Dex was instead apparently manifested through augmentation of NKp30-related NK cell functions. Despite low expression levels of NKp30 on stage IV NSCLC patient NK cells, NKp30-stimulated production of IFN- $\gamma$ and TNF- $\alpha$ by circulating NK cells was increased after four IFN- $\gamma$-Dex vaccinations, as compared with the start of the study. Importantly, this increased NKp30-elicited NK cell activation correlated with longer PFS (39).

With regard to a potential mechanism for Dex stimulation of NK cells, it was found that the Dex vaccine preparations possessed the aforementioned membrane-associated NKp30 ligand BAG6, which may have been responsible for activating NK cells of patients in an NKp30-dependent fashion. In support of this hypothesis, BAG6 levels correlated with the MHC-II concentrations of Dex inocula and NKp30-dependent NK cell functions. This differs from the finding of the previous MM phase I study in which NKG2D (and potentially IL-15/IL-15R $\alpha$ ) signaling was responsible for Dex-mediated NK cell activation $(37,42)$. Because the Dex immunotherapies utilized in this previous study were not derived from IFN- $\gamma$-mDCs in which IFN- $\gamma$ can upregulate BAG6 expression (51), NKG2D-L-mediated NK cell stimulation presumably featured more prominently in the absence of BAG6/NKp30 signaling.

\section{Future directions for Dex immunotherapies}

The early clinical testing of Dex as cancer vaccines has shown limited clinical efficacy in advanced cancer patient cohorts, although cases of disease stabilization were observed in each study. The limited efficacy in the trials might be explained by poor stimulation of adaptive immunity in these patients, potentially due to several factors. A conceivable explanation is the heterogeneity and small size of the patient cohorts, which had received previous anticancer treatments before enrollment. More importantly, systemic and local immunoregulatory mechanisms (e.g., programmed death ligand-1 [PD-L1] expression in NSCLC cells associated with programmed death-1 [PD-1] upregulation by tumor-infiltrating lymphocytes [TILs], Treg, and myeloid-derived suppressor cell [MDSC] activity) at play in these advanced-stage patients may have also significantly limited this immunotherapy. Another possibility is that the Dex MHC-Iand MHC-II-restricted antigens employed in these advanced patient cohorts were insufficient to drive tumor-targeted $\mathrm{T}$ cell responses. The potential of mutated neoepitopes to drive high-avidity T lymphocytes could be exploited for NSCLC patient exosomes $(52,53)$. Additionally, the in vivo trafficking and fate of Dex in patients is unknown. Instead of reaching the $\mathrm{T}$ cell zones of secondary lymphoid organs in sufficient numbers, Dex may be transported to subcapsular sinus macrophages or lymphatic sinus DCs to encounter innate lymphocytes (54). Lastly, it remains to be determined whether Dex express functional PD-L1 or PD-L2 molecules that may restrict $\mathrm{T}$ cell responses.

Future strategies for development of Dex as an immunotherapy. Given the problems encountered within a clinical setting, it will be necessary to develop strategies to enhance Dex activity to create a 
successful immunotherapy. Dex can be engineered to enhance particular immunostimulatory characteristics; therefore, it is certainly possible to further improve upon the most recent second-generation Dex. Engineering Dex to have a greater surface expression of various costimulatory molecules, with lower expression of immunoregulatory molecules (e.g., immune checkpoint molecules like PD-L1) may enhance Dex activity. The choice of TAAs adopted in the Dex clinical trials might also be improved upon, perhaps including several different TAAs or mutated neoantigens to combat tumor evolution $(2,53,55)$. Increasing the delivered quantity of such TAAs or the addition of GM-CSF to Dex immunotherapies may also help stimulate $\mathrm{T}$ cell responses, given the CTL activation observed in the Aex phase I trial (40). Moreover, recent findings have suggested that the design of Dex immunotherapies to stimulate $\mathrm{B}$ cell responses alongside $\mathrm{T}$ cell stimulation may optimize their immunogenicity $(56,57)$. Vaccination of mice with protein-loaded Dex, but not $\mathrm{T}$ cell peptide-loaded Dex, induced CD8 ${ }^{+}$ $\mathrm{T}$ cell responses and control of tumor growth, and these effects depended on the presence of both B cells and CD4 ${ }^{+} \mathrm{T}$ cells (56).

Dex immunotherapies may also benefit from the addition of TLR ligand adjuvants. CpG and polyinosinic:polycyctidylic acid - which activate TLR9 and TLR3, respectively - induced efficient tumor rejection and Dex-mediated $\mathrm{CD}^{+} \mathrm{T}$ cell priming in a melanoma model using HLA-A2 transgenic mice (58). Thus, the inherent capability of Dex to bind TLR ligands, thereby activating bystander DCs (59), could be implemented to enhance their immunogenicity. In addition to their immunostimulatory capacity, Dex therapies could be engineered to target tumor cells directly $(36,45)$. Indeed, expression of TNF, FasL, and TRAIL on Dex surface membranes directly triggers caspase activation and apoptosis in tumor cells (45). Engineering Dex to transfer mRNAs encoding relevant neoantigen peptides, or miRs or mRNAs that modulate distinct signaling pathways in immune cells or cancer cells, are other possibilities $(20,21,60,61)$.

Potential next steps for the clinical testing of Dex as an immunotherapy. Dex immunotherapy may be most effective as part of a combinatorial treatment regime. Firstly, combining CTX chemotherapy with Dex vaccines in vivo significantly enhanced tumoror peptide-induced $\mathrm{CD}^{+} \mathrm{T}$ cell recall responses, leading to a synergistic effect against preestablished tumors (47). However, CTX combination therapy only allowed efficient $\mathrm{T}$ cell priming by Dex in humans if genuine adjuvants were present. It is likely that the therapeutic effects seen in this scenario were due to a Dex/CTX therapy retuning of tumor-induced tolerance toward tumor-induced immunogenicity, since CTX can attenuate the suppressive function of Tregs $(47,50,62,63)$. The phase II Dex clinical trial similarly employed CTX to inhibit Tregs prior to Dex immunotherapy $(39,46)$, although the clinical efficacy of this combination would be more evident in a less advanced cohort. Secondly, combining Dex with PD-1/PD-L1 blockade (or coblockade with antiCTLA4 therapy) could reduce TIL suppression and enhance T cell priming. Thirdly, a major disadvantage of dealing with NSCLC patients is that the current standard chemotherapeutic regimen does not induce immunogenic cell death (64). Combining Dex with immunogenic cytotoxic drugs would be a suitable option in other malignancies $(65,66)$. Lastly, it is conceivable that Dex- and NK cell-based immunotherapies (such as anti-killer cell immuno- globulin-like receptor Ab [anti-KIR Ab]; refs. 67-69) may have synergistic effects against NK cell-dependent malignancies such as gastrointestinal stromal tumors, neuroblastomas, leukemias, and kidney cancers (70-72).

Notwithstanding the many strategies to develop Dex immunotherapy, the predominant reason for the limited efficacy of Dex immunotherapies achieved in clinical trials thus far could be due to the small, advanced cancer-stage patient cohorts used and to the lack of preselection criteria $(37,38)$. Nonetheless, our phase II Dex trial (39) revealed that patients with detectable serum BAG6 (most probably associated with NKp30 functional defects) are most likely to benefit from Dex therapy. Indeed, patients destined to best respond to Dex appear to be those presenting with defective NK cell receptor expression or functions (notably NKG2D or NKp30), with the predominantly targeted NK receptor dependent upon the specific Dex preparation (e.g., immature versus mature Dex) $(39,42)$. In prospective clinical studies, these parameters (e.g., killer activated receptors [KARs] and their circulating ligands) should be followed with comprehensive immunomonitoring analyses. Careful patient monitoring for potential immunerelated adverse events is also necessary in future trials involving Dex, despite Dex immunotherapy showing a strong safety profile thus far. This, alongside careful patient screening and selection, will be particularly important for future studies investigating potential combinatorial regimes with Dex (e.g., Dex combined with immune checkpoint inhibitors).

\section{Conclusions}

Despite the challenges encountered thus far, the prospect of Dex immunotherapy as a novel cancer treatment remains highly promising. Dex are adept mediators of immune responses and the relative technical ease in manipulating their immunostimulatory characteristics (via the donor DC), along with the advantages over cell-based therapies, ensures their future therapeutic uses. Although Dex immunotherapies produce an encouraging stimulatory effect upon NK cells, which likely contributed to the cases where improved clinical outcome was observed (39), it is clear that improved strategies to overcome the apparent lack of $\mathrm{T}$ cell responses are necessary. Focus on this hurdle, as well as on less understood Dex components and functions - such as mRNAs, miRs, transported cytokines, production of lipid mediators (73), and mechanisms through which each of these factors interact with acceptor immune cells - will help Dex immunotherapies to reach their full potential.

\section{Acknowledgments}

We thank Association de Recherche Contre le Cancer for supporting the phase II trial and J.M. Pitt. We are indebted to private philanthropy, namely Mrs. Badinter and Mrs. Agnès b. for their generous support of clinical trials. We thank N. Chaput and V. Lapierre for their work in the cell facility, as well as patients and their families for continuous support.

Address correspondence to: Laurence Zitvogel, Gustave Roussy Cancer Campus, 114 rue Edouard Vaillant, 94805 VILLEJUIF Cedex,France.Phone:33.1.42.11.50.41;E-mail:laurence.zitvogel@ gustaveroussy.fr. 
1. Steinman RM. Decisions about dendritic cells: past, present, and future. Annu Rev Immunol. 2012;30:1-22.

2. Chen DS, Mellman I. Oncology meets immunology: the cancer-immunity cycle. Immunity. 2013;39(1):1-10.

3. Kantoff PW, et al. Sipuleucel-T immunotherapy for castration-resistant prostate cancer. $N$ Engl J Med. 2010;363(5):411-422.

4. Pitt JM, et al. Dendritic cell-derived exosomes as immunotherapies in the fight against cancer. JImmunol. 2014;193(3):1006-1011.

5. Andre F, Escudier B, Angevin E, Tursz T, Zitvogel L. Exosomes for cancer immunotherapy. Ann Oncol. 2004;15(suppl 4):iv141-iv144.

6. Zhang B, Yin Y, Lai RC, Lim SK. Immunotherapeutic potential of extracellular vesicles. Front Immunol. 2014;5:518.

7. Gabrilovich DI, Ciernik IF, Carbone DP. Dendritic cells in antitumor immune responses. I. Defective antigen presentation in tumor-bearing hosts. Cell Immunol. 1996;170(1):101-110.

8. Melo SA, et al. Glypican-1 identifies cancer exosomes and detects early pancreatic cancer. Nature. 2015;523(7559):177-182.

9. Zijlstra C, Stoorvogel W. Prostasomes as a source of diagnostic biomarkers for prostate cancer. JClin Invest. 2016;126(4):1144-1151.

10. Kosaka N, Yoshioka Y, Fujita Y, Ochiya T. Versatile roles of extracellular vesicles in cancer.JClin Invest. 2016;126(4):1163-1172.

11. Whiteside TL. Exosomes and tumor-mediated immune suppression. J Clin Invest. 2016;126(4):1216-1223.

12. Thery C, Ostrowski M, Segura E. Membrane vesicles as conveyors of immune responses. Nat Rev Immunol. 2009;9(8):581-593.

13. Harding CV, Heuser JE, Stahl PD. Exosomes: looking back three decades and into the future. JCell Biol. 2013;200(4):367-371.

14. Robbins PD, Morelli AE. Regulation of immune responses by extracellular vesicles. Nat Rev Immunol. 2014;14(3):195-208.

15. Thery C, et al. Proteomic analysis of dendritic cell-derived exosomes: a secreted subcellular compartment distinct from apoptotic vesicles. JImmunol. 2001;166(12):7309-7318.

16. Thery C, et al. Molecular characterization of dendritic cell-derived exosomes. Selective accumulation of the heat shock protein hsc73. J Cell Biol. 1999;147(3):599-610.

17. Zitvogel L, et al. Eradication of established murine tumors using a novel cell-free vaccine: dendritic cell-derived exosomes. Nat Med. 1998;4(5):594-600.

18. Tamura Y, et al. Heat-shock proteins as endogenous ligands building a bridge between innate and adaptive immunity. Immunotherapy. 2012;4(8):841-852.

19. Valadi H, Ekstrom K, Bossios A, Sjostrand M, Lee JJ, Lotvall JO. Exosome-mediated transfer of mRNAs and microRNAs is a novel mechanism of genetic exchange between cells. Nat Cell Biol. 2007;9(6):654-659.

20. Zhang J, et al. Exosome and exosomal microRNA: trafficking, sorting, and function. Genomics Proteomics Bioinformatics. 2015;13(1):17-24.

21. Montecalvo A, et al. Mechanism of transfer of functional microRNAs between mouse dendritic cells via exosomes. Blood. 2012;119(3):756-766.

22. Raposo G, et al. B lymphocytes secrete antigen-presenting vesicles. J Exp Med. 1996;183(3):1161-1172.

23. Admyre C, Johansson SM, Paulie S, Gabrielsson S. Direct exosome stimulation of peripheral human T cells detected by ELISPOT. Eur J Immunol.2006;36(7):1772-1781.

24. Utsugi-Kobukai S, Fujimaki H, Hotta C, Nakazawa M, Minami M. MHC class I-mediated exogenous antigen presentation by exosomes secreted from immature and mature bone marrow derived dendritic cells. Immunol Lett. 2003;89(2-3):125-131.

25. Muntasell A, Berger AC, Roche PA. T cellinduced secretion of MHC class II-peptide complexes on B cell exosomes. EMBO J. 2007;26(19):4263-4272.

26. Montecalvo A, et al. Exosomes as a short-range mechanism to spread alloantigen between dendritic cells during $\mathrm{T}$ cell allorecognition. J Immunol. 2008;180(5):3081-3090.

27. Vincent-Schneider H, et al. Exosomes bearing HLA-DR1 molecules need dendritic cells to efficiently stimulate specific $\mathrm{T}$ cells. Int Immunol. 2002;14(7):713-722.

28. Thery C, Duban L, Segura E, Veron P, Lantz O, Amigorena S. Indirect activation of naive $\mathrm{CD} 4^{+}$ $\mathrm{T}$ cells by dendritic cell-derived exosomes. $\mathrm{Nat}$ Immunol. 2002;3(12):1156-1162.

29. Andre F, et al. Exosomes as potent cell-free peptide-based vaccine. I. Dendritic cell-derived exosomes transfer functional MHC class I/ peptide complexes to dendritic cells. JImmunol. 2004;172(4):2126-2136.

30. Wakim LM, Bevan MJ. Cross-dressed dendritic cells drive memory $\mathrm{CD} 8^{+} \mathrm{T}$-cell activation after viral infection. Nature. 2011;471(7340):629-632

31. Viaud S, et al. Updated technology to produce highly immunogenic dendritic cell-derived exosomes of clinical grade: a critical role of interferon- $\gamma$. J Immunother. 2011;34(1):65-75.

32. Segura E, Amigorena S, Thery C. Mature dendritic cells secrete exosomes with strong ability to induce antigen-specific effector immune responses. Blood Cells Mol Dis. 2005;35(2):89-93.

33. Segura $\mathrm{E}$, et al. ICAM-1 on exosomes from mature dendritic cells is critical for efficient naive T-cell priming. Blood. 2005;106(1):216-223.

34. Mallegol J, et al. T84-intestinal epithelial exosomes bear MHC class II/peptide complexes potentiating antigen presentation by dendritic cells. Gastroenterology. 2007;132(5):1866-1876.

35. Nakayama M. Antigen presentation by MHC dressed cells. Front Immunol. 2014;5:672.

36. Romagnoli GG, Zelante BB, Toniolo PA, Migliori IK, Barbuto JA. Dendritic cell-derived exosomes may be a tool for cancer immunotherapy by converting tumor cells into immunogenic targets. Front Immunol. 2014;5:692.

37. Escudier B, et al. Vaccination of metastatic melanoma patients with autologous dendritic cell (DC) derived-exosomes: results of thefirst phase I clinical trial. J Transl Med. 2005;3(1):10.

38. Morse MA, et al. A phase I study of dexosome immunotherapy in patients with advanced nonsmall cell lung cancer. J Transl Med. 2005;3(1):9.

39. Besse B, et al. Dendritic cell-derived exosomes as maintenance immunotherapy after first line chemotherapy in NSCLC [published online ahead of print February 2, 2016]. Oncoimmunology. doi:10. 1080/2162402X.2015.1071008.

40. Dai S, et al. Phase I clinical trial of autologous ascites-derived exosomes combined with GM-CSF for colorectal cancer. Mol Ther. 2008;16(4):782-790.

41. Yang C, Robbins PD. The roles of tumor-derived exosomes in cancer pathogenesis. Clin Dev Immunol. 2011;2011:842849.

42. Viaud S, et al. Dendritic cell-derived exosomes promote natural killer cell activation and proliferation: a role for NKG2D ligands and IL-15Ralpha. PLoS One. 2009;4(3):e4942.

43. Simhadri VR, et al. Dendritic cells release HLA-B-associated transcript-3 positive exosomes to regulate natural killer function. PLoS One. 2008;3(10):e3377.

44. Pogge von Strandmann E, et al. Human leukocyte antigen-B-associated transcript 3 is released from tumor cells and engages the $\mathrm{NKp} 30$ receptor on natural killer cells. Immunity. 2007;27(6):965-974.

45. Munich S, Sobo-Vujanovic A, Buchser WJ, BeerStolz D, Vujanovic NL. Dendritic cell exosomes directly kill tumor cells and activate natural killer cells via TNF superfamily ligands. Oncoimmunology. 2012;1(7):1074-1083.

46. Viaud S, et al. Dendritic cell-derived exosomes for cancer immunotherapy: what's next? Cancer Res. 2010;70(4):1281-1285.

47. Taieb J, et al. Chemoimmunotherapy of tumors: cyclophosphamide synergizes with exosome based vaccines. JImmunol. 2006;176(5):2722-2729.

48. Viaud S, et al. Cyclophosphamide induces differentiation of Th17 cells in cancer patients. Cancer Res. 2011;71(3):661-665.

49. Viaud S, et al. The intestinal microbiota modulates the anticancer immune effects of cyclophosphamide. Science. 2013;342(6161):971-976.

50. Ghiringhelli F, et al. Metronomic cyclophosphamide regimen selectively depletes $\mathrm{CD} 4{ }^{+} \mathrm{CD} 25^{+}$regulatory $\mathrm{T}$ cells and restores $\mathrm{T}$ and NK effector functions in end stage cancer patients. Cancer Immunol Immunother. 2007;56(5):641-648.

51. Kamper N, Franken S, Temme S, Koch S, Bieber T, Koch N. gamma-Interferon-regulated chaperone governs human lymphocyte antigen class II expression. FASEB J. 2012;26(1):104-116.

52. Kreiter S, et al. Mutant MHC class II epitopes drive therapeutic immune responses to cancer. Nature. 2015;520(7549):692-696.

53. Schumacher TN, Schreiber RD. Neoantigens in cancer immunotherapy. Science. 2015;348(6230):69-74

54. Germain RN, et al. Making friends in out-of-theway places: how cells of the immune system get together and how they conduct their business as revealed by intravital imaging. Immunol Rev. 2008;221:163-181.

55. Schreiber RD, Old LJ, Smyth MJ. Cancer immunoediting: integrating immunity's roles in cancer suppression and promotion. Science. 2011;331(6024):1565-1570.

56. Naslund TI, Gehrmann U, Qazi KR, Karlsson MC, Gabrielsson S. Dendritic cell-derived exosomes 
need to activate both $\mathrm{T}$ and $\mathrm{B}$ cells to induce antitumor immunity. J Immunol. 2013;190(6):2712-2719.

57. Qazi KR, Gehrmann U, Domange Jordo E, Karlsson MC, Gabrielsson S. Antigen-loaded exosomes alone induce Th1-type memory through a B-cell-dependent mechanism. Blood. 2009;113(12):2673-2683.

58. Chaput N, et al. Exosomes as potent cell-free peptide-based vaccine. II. Exosomes in CpG adjuvants efficiently prime naive Tc1 lymphocytes leading to tumor rejection. JImmunol. 2004;172(4):2137-2146.

59. Sobo-Vujanovic A, Munich S, Vujanovic NL. Dendritic-cell exosomes cross-present Toll-like receptor-ligands and activate bystander dendritic cells. Cell Immunol. 2014;289(1-2):119-127.

60. Dreux M, et al. Short-range exosomal transfer of viral RNA from infected cells to plasmacytoid dendritic cells triggers innate immunity. Cell Host Microbe. 2012;12(4):558-570.

61. Melo SA, et al. Cancer exosomes perform cell-independent microRNA biogenesis and promote tumorigenesis. Cancer Cell. 2014;26(5):707-721.

62. Ghiringhelli $\mathrm{F}$, et al. $\mathrm{CD} 4{ }^{+} \mathrm{CD} 25^{+}$regulatory $\mathrm{T}$ cells suppress tumor immunity but are sensitive to cyclophosphamide which allows immunotherapy of established tumors to be curative. Eur J
Immunol. 2004;34(2):336-344.

63. Hermans IF, Chong TW, Palmowski MJ, Harris AL, Cerundolo V. Synergistic effect of metronomic dosing of cyclophosphamide combined with specific antitumor immunotherapy in a murine melanoma model. Cancer Res. 2003;63(23):8408-8413.

64. Vacchelli E, et al. Trial Watch: Chemotherapy with immunogenic cell death inducers. Oncoimmunology. 2014;3(1):e27878.

65. Kroemer G, Galluzzi L, Kepp O, Zitvogel L. Immunogenic cell death in cancer therapy. Annu Rev Immunol. 2013;31:51-72.

66. Zitvogel L, Galluzzi L, Smyth MJ, Kroemer G. Mechanism of action of conventional and targeted anticancer therapies: reinstating immunosurveillance. Immunity. 2013;39(1):74-88.

67. Alici E. IPH-2101, a fully human anti-NK-cell inhibitory receptor $\mathrm{mAb}$ for the potential treatment of hematological cancers. Curr Opin Mol Ther. 2010;12(6):724-733.

68. Benson DM Jr, et al. IPH2101, a novel anti-inhibitory KIR antibody, and lenalidomide combine to enhance the natural killer cell versus multiple myeloma effect. Blood. 2011;118(24):6387-6391.

69. Marie-Cardine A, et al. IPH4102, a humanized KIR3DL2 antibody with potent activity against cutaneous T-cell lymphoma. Cancer Res.
2014;74(21):6060-6070.

70. Semeraro M, et al. Clinical impact of the NKp3O B7-H6 axis in high-risk neuroblastoma patients. Sci Transl Med. 2015;7(283):283ra55.

71. Rusakiewicz S, et al. Immune infiltrates are prognostic factors in localized gastrointestinal stromal tumors. Cancer Res. 2013;73(12):3499-3510.

72. Remark R, et al. Characteristics and clinical impacts of the immune environments in colorectal and renal cell carcinoma lung metastases: influence of tumor origin. Clin Cancer Res. 2013;19(15):4079-4091.

73. Esser J, et al. Exosomes from human macrophages and dendritic cells contain enzymes for leukotriene biosynthesis and promote granulocyte migration. J Allergy Clin Immunol. 2010;126(5):1032-1040.

74. Bajenoff M, et al. Natural killer cell behavior in lymph nodes revealed by static and real-time imaging. J Exp Med. 2006;203(3):619-631.

75. Saunderson SC, Dunn AC, Crocker PR, McLellan AD. CD169 mediates the capture of exosomes in spleen and lymph node. Blood. 2014;123(2):208-216.

76. Wiklander OP, et al. Extracellular vesicle in vivo biodistribution is determined by cell source, route of administration and targeting. J Extracell Vesicles. 2015;4:26316. 